\title{
Hospital-based evaluation of tuberculosis-exposed neonates: An approach to complement the South African national guidance
}

\author{
A van der Westhuizen, ${ }^{1} \mathrm{MB}$ ChB; A Dramowski, ${ }^{2} \mathrm{MB}$ ChB, FC Paed (SA), MMed (Paed), Cert Paed ID, PhD; H S Schaaf, ${ }^{2} \mathrm{MB}$ ChB, \\ MMed (Paed), PhD; M Groenewald, ${ }^{3}$ MB ChB; A Bekker, ${ }^{2,3}$ MB ChB, FC Paed (SA), MMed (Paed), Cert Neonatology (SA), PhD \\ ${ }^{1}$ Frere and Cecilia Makiwane hospitals, East London, South Africa \\ ${ }^{2}$ Department of Paediatrics and Child Health, Faculty of Medicine and Health Sciences, Stellenbosch University and Tygerberg Hospital, \\ Cape Town, South Africa \\ ${ }^{3}$ Family Centre for Research with Ubuntu, Department of Paediatrics and Child Health, Faculty of Medicine and Health Sciences, Stellenbosch \\ University, Cape Town, South Africa
}

Corresponding author: A Bekker (adrie@sun.ac.za)

The South African National Department of Health published updated guidelines in 2019 for the prevention of mother-to-child transmission of communicable diseases. The proposed management of a neonate born to a mother with tuberculosis (TB) was included, and recommended referral of all symptomatic TB-exposed neonates to hospital for TB evaluation. However, no standard approach exists for evaluating hospitalised, symptomatic TB-exposed neonates, including preterm and low-birthweight (LBW) neonates born to mothers with TB. We use a clinical case report to illustrate a suggested approach to hospital-based evaluation of TB-exposed neonates, including preterm and LBW neonates. Guidance for the interpretation of different TB screening investigations in this population is also provided.

S Afr Med J 2021;111(8):724-728. https://doi.org/10.7196/SAMJ.2021.v111i8.15575

Tuberculosis (TB) is the leading non-obstetric cause of maternal death in South Africa (SA), and is often difficult to diagnose during pregnancy. ${ }^{[1-3]}$ Ante- and intrapartum transmission of Mycobacterium tuberculosis occurs haematogenously via the placenta or when infected amniotic fluid is aspirated or ingested during delivery, and may result in congenital TB. Although congenital transmission is uncommon, it is more likely to occur when the mother has primary TB or disseminated disease. ${ }^{[4]}$ Postnatal TB transmission via respiratory airborne spread is more common when she has adult-type (cavitary) pulmonary TB. ${ }^{[4]}$ The screening investigations, diagnosis and treatment of term and preterm neonates with possible or confirmed congenital or postnatal TB are the same, independent of the timing of $M$. tuberculosis infection.

$\mathrm{TB}$ in pregnancy is associated with unfavourable outcomes for both mothers and their infants, with double the risk of preterm delivery ( $<37$ weeks' gestational age) and/or low birthweight (LBW) $(<2500 \mathrm{~g})$ compared with healthy mothers. ${ }^{[5]}$ The diagnosis of TB in neonates is challenging, particularly in preterm and LBW infants, in whom respiratory symptoms due to hyaline membrane disease or other causes can be difficult to distinguish from pneumonia caused by TB. Neonates born to mothers with TB, i.e. TB-exposed neonates, can be asymptomatic, clinically well, or present with nonspecific signs such as fever, respiratory distress, abdominal distension from ascites and/or hepatosplenomegaly, or even obstructive jaundice. ${ }^{[6-8]}$ Without preventive therapy, up to $50 \%$ of TB-exposed infants $(<12$ months of age) will develop TB disease, with disseminated or progressive pulmonary disease in $30 \%$ of these cases. ${ }^{[9]}$ Appropriate assessment and management of the TB-exposed neonate is therefore essential, given the high risk of progression from TB infection to TB disease in this population.
In 2019, the SA National Department of Health (NDoH) published updated guidelines for the prevention of mother-to-child transmission of communicable diseases. ${ }^{[10]}$ These guidelines included an algorithm describing the management of any TB-exposed neonate born to a mother who may pose a TB infectious risk to her newborn baby. This was defined as a mother with $\mathrm{TB}$ diagnosed in the last 2 months of pregnancy or a mother who has not responded to $\mathrm{TB}$ treatment or whose sputum remains positive for acid-fast bacilli (AFB). The focus of the $\mathrm{NDoH}$ guidance is mainly on term neonates born at midwife obstetric units, and it recommends referral of all symptomatic TB-exposed neonates (respiratory rate $>60 /$ min, difficulty in breathing, feeding problems, poor weight gain, abdominal distension, enlarged liver/spleen and/or jaundice) to hospital for evaluation for TB. The $\mathrm{NDoH}$ guideline does not provide recommendations on the hospital-based investigation and management of these referred, symptomatic, TB-exposed neonates, nor does it provide an approach to evaluation of hospitalised preterm and LBW TB-exposed neonates. Investigations for and treatment of $\mathrm{TB}$ are similar for term and preterm neonates, but a low threshold for investigating preterm TB-exposed infants is essential owing to frequent atypical presentations and a high risk of progression of disease. $^{[4]}$

No standard approach exists for the evaluation of hospitalised symptomatic term and symptomatic or asymptomatic preterm and/ or LBW TB-exposed neonates, with practices varying greatly. ${ }^{[7]}$ Given the diagnostic challenges, we use a case study to illustrate the different TB screening investigations available for TB-exposed neonates and the interpretation thereof. Furthermore, we propose a hospital-based evaluation algorithm for the TB-exposed neonate to complement the $\mathrm{NDoH}$ guidelines. 


\section{Case study}

A 30-year-old woman presented to Tygerberg Hospital, Cape Town, $\mathrm{SA}$, in spontaneous preterm labour (SPTL) with prolonged rupture of membranes (PRoM), at an estimated 33 weeks' gestation. She had received no antenatal care, and admission screening tests for HIV and syphilis were negative. She gave birth to a preterm, LBW (2 252 g) male infant via normal vertex delivery. Although the neonate appeared well after delivery, he was admitted for investigation of possible neonatal sepsis because of the maternal risk factors of SPTL and PRoM, and was initiated on prophylactic ampicillin and gentamicin. While visiting her baby, the mother complained of a persistent cough and night sweats. She reported submitting a sputum sample for TB investigation at her local clinic a few days before delivery. She had previously been treated for drugsusceptible pulmonary TB (DS-TB) in 2014 and 2016, but reported treatment interruption after 3 months in the latter episode. Her current sputum samples were both smear-negative for AFB with M. tuberculosis cultures pending. Her chest radiograph showed cavitary lung disease with fibrosis.

Given the high risk of perinatal TB disease in this hospitalised preterm LBW infant, born to a mother with current symptoms of $\mathrm{TB}$ and a history of previously interrupted TB treatment, a decision was made to assess the neonate for TB. The TB evaluation included clinical examination, radiological imaging and laboratory investigations. The findings on clinical examination and the chest radiograph were normal. Two gastric aspirates were sent for smear microscopy for AFB, Xpert MTB/RIF Ultra (Xpert Ultra) and mycobacterial culture. The smear microscopy for AFB was negative, but Xpert Ultra was trace positive for $M$. tuberculosis complex on one gastric aspirate taken on day 2 after birth (rifampicin susceptibility testing was unsuccessful). After consultation with paediatric infectious disease experts, an abdominal ultrasound scan was requested to investigate for possible hepatic lesions suggestive of TB; this was normal. Subsequently, a decision was made not to treat this asymptomatic, TB-exposed neonate, but rather to give him TB preventive therapy (TPT) (as low amounts of M. tuberculosis DNA in gastric aspirates may represent bacilli ingested in utero or at birth). In the light of the normal findings on clinical examination and the normal chest radiograph and abdominal ultrasound scan, isoniazid was commenced as TPT on day 5 after birth at a dose of $10 \mathrm{mg} / \mathrm{kg} / \mathrm{d}$. Isoniazid therapy was deemed sufficient to eradicate the ingested bacilli and prevent TB disease progression. The infant was discharged into the care of his mother, who was restarted on DS-TB treatment by the local clinic based on her clinical features and the infant's Xpert Ultra results. BCG vaccination was deferred until after completion of TPT. Regular outpatient follow-up visits were scheduled to monitor the infant's wellbeing and growth, with several dose adjustments needed (a three-fold increase in isoniazid dose from initiation to completion of TPT). All M. tuberculosis cultures from the neonate's specimens were negative. The infant and mother remained well, and both had successfully completed TPT and DS-TB therapy, respectively, at the 6-month visit. BCG vaccination was given after completion of TPT and no tuberculin skin test (TST) was performed.

This case study illustrates an asymptomatic but preterm and LBW TB-exposed neonate who was considered to be at high risk of TB infection because of the history of untreated TB in a symptomatic mother. Clinical examination and screening investigations for TB in this neonate were negative, except for one trace-positive Xpert Ultra gastric aspirate result, which probably reflected TB bacilli ingested at birth. The neonate was accordingly assessed as not having TB disease and was therefore commenced on a course of TPT only, with regular clinical follow-up to evaluate for the development of any TB symptoms and/or signs. The positive Xpert Ultra result in the neonate facilitated rapid initiation of TPT, and TB disease was successfully prevented. In addition, the laboratory confirmation of TB led to the rapid recommencement of TB treatment in the mother, who had a history of chronic symptoms and a chest radiograph with bilateral upper-lobe adult-type cavitary TB.

\section{Ethics approval and informed consent}

Written informed consent for the publication of this case report was obtained from the patient, and ethical approval was obtained from the Stellenbosch University Faculty of Medicine and Health Sciences Undergraduate Research Ethics Committee (ref. no. UC19/08/032).

\section{Discussion and recommendations}

The $2019 \mathrm{NDoH}$ guidance ${ }^{[10]}$ provides recommendations for the management of asymptomatic TB-exposed infants and instructs that symptomatic neonates be referred to hospital for TB evaluation. Information on how this evaluation should be conducted is lacking, however, with no guidance offered on how to manage preterm and LBW TB-exposed neonates, which we suggest should be included in the hospital-based evaluation of a TB-exposed neonate. To our knowledge, no standard approach for the evaluation of symptomatic TB-exposed neonates exists. A review by Mittal et al. ${ }^{[7]}$ tried to capture the varying approaches to the management of TB-exposed neonates, which are mainly based on expert opinion.

To address the lack of clinical guidance for in-hospital management of TB-exposed neonates, we propose an evaluation algorithm that includes both symptomatic term neonates and asymptomatic and symptomatic preterm and LBW neonates (Fig. 1). At Tygerberg Hospital, an academic referral hospital in Cape Town, SA, all symptomatic TB-exposed neonates, including all TB-exposed preterm and/or LBW neonates, are evaluated for TB disease with a minimum of two gastric aspirates for AFB smear microscopy, mycobacterial culture and Xpert Ultra, and a chest radiograph. Depending on the clinical symptoms and signs, other directed investigations may also be appropriate (Fig. 1 and Table 1).

Once TB disease has been excluded in a symptomatic term neonate born to a mother with infectious TB (newly diagnosed, treated for $<2$ months, still culture-positive or AFB smear-positive), TPT is initiated. We also make use of the same strategy when managing asymptomatic or symptomatic preterm or LBW neonates born to an infectious mother with TB. The World Health Organization (WHO) recommends either 6 months of isoniazid or 3 months of isoniazid plus rifampicin (Tables 2 and 3 ) for the prevention of DS-TB. ${ }^{[11]}$ Regular (monthly) follow-up visits should be scheduled for all TB-exposed neonates to record weight gain and adjust the TPT medication doses, assess infants for new TB symptoms or signs, and if these are present, re-evaluate for TB disease.

In symptomatic, preterm or LBW infants born in SA where TB evaluation confirms or is suggestive of $\mathrm{TB}$ disease, a four-drug TB treatment should be started immediately (Tables 2 and 3). As recommended by the WHO, children with probable or confirmed pulmonary $\mathrm{TB}$ or $\mathrm{TB}$ peripheral lymphadenitis and/or children with extensive pulmonary disease, living in settings where the prevalence of HIV is high and/or the prevalence of isoniazid resistance is high, should be treated with a four-drug regimen for 2 months followed by a two-drug regimen for 4 months. ${ }^{[12]}$ Owing to the difficulty in monitoring for ethambutol-induced ocular toxicity in neonates, ethionamide is sometimes preferred as the fourth drug, with the added advantage of good central nervous system 


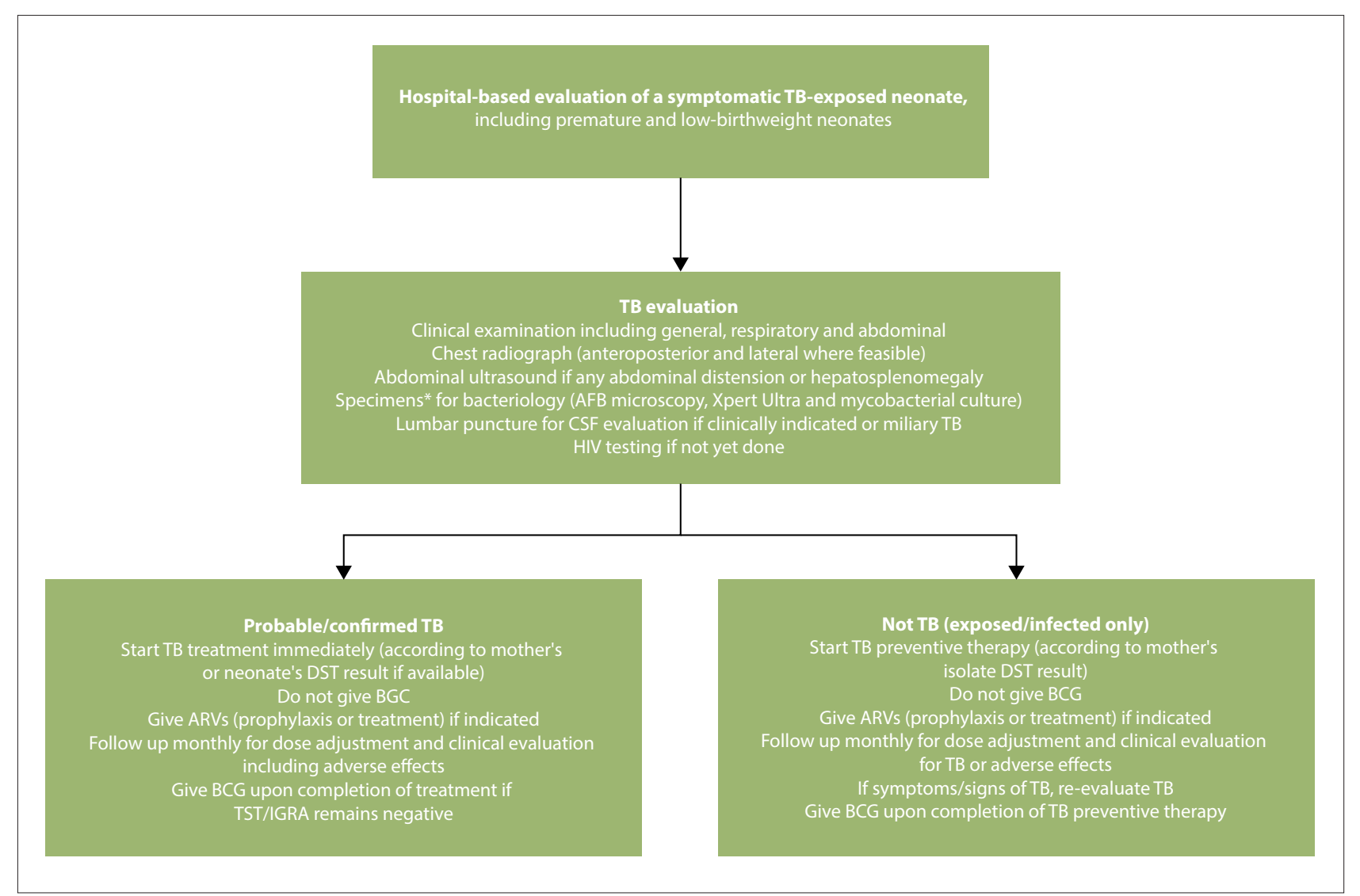

Fig. 1. Algorithm for hospital-based evaluation of a symptomatic neonate exposed to maternal $T B$. $(T B=$ tuberculosis; $A F B=$ acid-fast bacilli; Xpert Ultra $=$ Xpert MTB/RIF Ultra; CSF = cerebrospinal fluid; DST = drug susceptibility testing; ARVs = antiretrovirals; TST = tuberculin skin test; IGRA = interferongamma release assay; ${ }^{*}$ Usually gastric or tracheal aspirates, the latter if intubated.)

penetration. ${ }^{[13,14]}$ The continuation phase of isoniazid and rifampicin (usually 4 months) may be prolonged to 7 months depending on the infant's initial disease severity and/or clinical response. ${ }^{[12]}$

In the case of asymptomatic neonates whose mothers with TB are no longer infectious (i.e. on effective treatment for $>2$ months and preferably sputum culture-negative), we advise that these neonates undergo a clinical examination; if the findings are normal, no TB screening tests are performed and no TPT is required. BCG should be administered immediately after birth. The neonate should be followed up regularly to monitor weight gain and evaluated for new TB symptoms or signs, which, if present, should lead to TB evaluation. The mother and/or other household members' TB treatment progress should be monitored, as the infant may be re-exposed if the TB-infected adult/s are lost to follow-up or experience treatment failure.

\section{BCG, HIV and drug-resistant TB considerations for TB-exposed neonates}

BCG vaccination should be deferred until 2 weeks after completion of TPT or anti-TB treatment, as anti-TB medications may kill the live BCG vaccine. The rationale for administering BCG following preventive or $\mathrm{TB}$ treatment is to protect against disseminated TB disease in the case of TB re-exposure. ${ }^{[15]}$ BCG is routinely administered at birth to neonates in SA, including those born to women living with HIV, often prior to receipt of the neonate's HIV PCR status. Universal early initiation of antiretroviral therapy in neonates with HIV may currently be the most effective strategy to reduce the risk of both BCG and TB disease in this population. ${ }^{[16]}$
HIV co-infection is not uncommon in pregnant women with TB. ${ }^{[17]}$ For HIV-exposed neonates receiving nevirapine as part of prevention of mother-to-child transmission of HIV treatment and requiring TB preventive therapy, TPT with isoniazid for 6 months is preferred. Prevention of TB using rifampicin plus isoniazid reduces nevirapine concentrations in HIV-exposed infants, potentially providing inadequate HIV preventive therapy with a risk of HIV seroconversion. ${ }^{[18]} \mathrm{An}$ isoniazid dose of $10 \mathrm{mg} / \mathrm{kg} / \mathrm{d}$ in LBW neonates was shown to achieve comparable adult target isoniazid plasma concentrations, cautioning against the use of higher isoniazid doses in smaller and younger infants with reduced drug elimination. ${ }^{[19]}$

The management of neonates exposed to drug-resistant TB should always be discussed with an expert, as although the same management principles apply, the anti-TB medications for preventive therapy and treatment differ. In the case of isoniazid mono-resistance, rifampicin for 4 months should be used as TPT. For rifampicin mono-resistant TB exposure when the source case's isolate exhibits phenotypic susceptibility to isoniazid, isoniazid preventive therapy for 6 months should be used; however, with rifampicin-resistant TB and unknown or unconfirmed isoniazid susceptibility or multidrugresistant $\mathrm{TB}$ exposure, an expert should be consulted. TB treatment in the neonate with drug-resistant TB disease should be tailored to the drug susceptibility test (DST) results of the mother's (source case's) M. tuberculosis isolate; DST should be done on the neonate's specimen if this is bacteriologically positive. If the mother's DST results are not available, drug-susceptible preventive therapy or treatment as appropriate should be started, and bacteriological results of both mother's and infant's specimens followed up. 


\section{Table 1. Guidance for the interpretation of hospital-based TB screening investigations}

Chest radiograph
Bacteriological confirmation
(respiratory specimens such as gastric
aspirates, tracheal aspirates) and other
relevant specimens
Abdominal ultrasound

Lumbar puncture for CSF evaluation

Other investigations

Placenta

Endometrial sampling

TST (Mantoux)

Interferon-gamma release assays (Quantiferon-TB Gold In-Tube or T-SPOT.TB)

Stool sample for Xpert MTB/RIF Ultra
Should be done regardless of whether symptoms or signs are present. Congenital TB may differ from postnatal TB on chest radiograph. ${ }^{[4]}$ Mediastinal nodes, airway compression, bronchopneumonic parenchymal infiltrates or a miliary pattern may occur. Also consider TB if pneumonic infiltrates do not respond to antibiotics.

Smear microscopy for AFB may be of value in neonates who aspirated TB-infected amniotic fluid or in infants with uncontained pulmonary TB. Xpert MTB/RIF Ultra is a more sensitive test than microscopy and may provide an additional RIF DST result. Mycobacterial culture, although slow, is currently still the most sensitive bacteriological test, and important for DST.

If abdominal distension or hepatosplenomegaly is present in the absence of any other confirmatory TB test, an abdominal ultrasound scan to look for focal lesions in the liver or spleen, or retroperitoneal or intra-abdominal lymphadenopathy, as well as ascites, can help to confirm a diagnosis of perinatal TB. ${ }^{[6]}$ CSF is often obtained in neonates as part of a septic work-up in early-onset sepsis with a positive blood culture or when meningitis is suspected; in the case of a lymphocytic predominance, TB meningitis should be considered. ${ }^{[21]}$ If a miliary picture is present on the chest radiograph, a lumbar puncture should be done.

Histopathological evaluation of placental TB, if the mother is not known to have had TB disease during the last trimester of pregnancy, may assist in the diagnosis of TB in the mother. ${ }^{[2]}$

Culture and histological analysis of a maternal endometrial pipelle sample for mycobacteria may be done to exclude genital tuberculosis or confirm congenital TB. ${ }^{[23]}$

Utility of TST in neonates is poor owing to low reactogenicity and poor helper T-cell responses. A positive TST is supportive evidence of TB infection or disease, but negative results do not rule out the possibility of TB infection or disease. ${ }^{[7]}$

Blood tests to confirm TB infection, like TST, are not very sensitive in neonates. They have the advantage of being more specific than TST, especially if BCG has been administered. ${ }^{[2]}$

Detecting Mycobacterium tuberculosis in stool samples of children using molecular methods is a promising non-invasive way of diagnosing pulmonary TB. Limitations include a lack of data on the performance of this method in infants and young children. ${ }^{[25]}$

$\mathrm{TB}=$ tuberculosis; $\mathrm{AFB}=$ acid-fast bacilli; $\mathrm{RIF}=$ rifampicin; $\mathrm{DST}=$ drug susceptibility testing; $\mathrm{CSF}=$ cerebrospinal fluid; $\mathrm{TST}$ = tuberculin skin test.

Table 2. Anti-TB regimens for neonates exposed to maternal drug-susceptible tuberculosis

\begin{tabular}{ll}
\hline & Anti-TB drug(s) \\
\hline TB preventive therapy & Isoniazid for 6 months, or \\
& Isoniazid plus rifampicin for 3 months \\
TB treatment & Intensive phase \\
& Isoniazid, rifampicin, pyrazinamide, ethambutol for 2 months, or \\
& Isoniazid, rifampicin, pyrazinamide, ethionamide for 2 months \\
& Continuation phase \\
& Isoniazid and rifampicin for $4-7$ months depending on disease severity and response to treatment
\end{tabular}

$\mathrm{TB}=$ tuberculosis

\section{Infection control measures for hospitalised TB-exposed neonates}

Mothers with TB and their newborns should preferably not be separated from each other in the hospital setting, although the mother should be isolated with her newborn in the same room if both are clinically stable; however, the mother should not be allowed in a neonatal ward while infectious. TB treatment should be started in the mother, and the neonate should receive preventive therapy or treatment according to the clinical decision. Breastfeeding should be encouraged, with the mother wearing a face cover if she is still infectious. ${ }^{[12]}$ The risk of TB transmission through breastmilk is negligible, and insignificant amounts of anti-TB medications are excreted in breastmilk, although data for the newer anti-TB drugs are
Table 3. Anti-TB drug dosages for neonates

\begin{tabular}{ll}
\hline Anti-TB drugs & Daily dose range $\mathbf{( m g} / \mathbf{k g})$ \\
\hline Isoniazid & $7-15$ \\
Rifampicin & $10-20$ \\
Pyrazinamide & $30-40$ \\
Ethambutol & $15-25$ \\
Ethionamide & $15-20$ \\
TB = tuberculosis. &
\end{tabular}

awaited. In high TB-burden settings, a TB screening policy should be in place where mothers share facilities, such as Kangaroo Mother 
Care units, to prevent exposure and transmission of TB to vulnerable neonates, mothers and healthcare workers. ${ }^{[20]}$

\section{Conclusions}

There are many challenges in the identification, investigation and clinical management of TB-exposed neonates. We propose an investigation and treatment algorithm for the hospital-based evaluation of symptomatic TB-exposed neonates, including preterm and LBW neonates, to guide clinicians to the earlier identification and diagnosis of TB infection and disease in SA neonatal practice.

\section{Declaration. None.}

Acknowledgements. We thank the mother of the infant described in this case report for providing consent for publication, and the Tygerberg neonatal service and the staff at the Family Centre for Research with Ubuntu (FAMCRU) for providing follow-up care of the infant.

Author contributions. AvdW, $\mathrm{AB}$ and $\mathrm{AD}$ drafted the first version of the manuscript. MG conducted the follow-up care of the infant at FAMCRU and reviewed the manuscript. AB, HSS and AD supervised the management of the infant. All authors gave critical input and approved the final version of the manuscript.

Funding. None.

Conflicts of interest. None.

1. National Committee on Confidential Enquiries into Maternal Deaths. Saving mothers 2014 - 2016 Seventh triennial report on confidential enquiries into maternal deaths in South Africa. Pretoria: National Department of Health, 2018. https://anaesthetics.ukzn.ac.za/Libraries/Obstets3 2019/ Saving_Mothers.pdf (accessed 4 June 2021)

. Moodley J, Fawcus S, Pattinson R. Improvements in maternal mortality in South Africa. S Afr Med J 2016;108(3, Suppl 1):S4-S8. https://doi.org/10.7196/SAMJ.2016.v106i5.10821

3. Hoffmann CJ, Variava E, Rakgokong M, et al. High prevalence of pulmonary tuberculosis but low sensitivity of symptom screening among HIV-infected pregnant women in South Africa. PLoS ONE 2013;8(4):e62211. https://doi.org/10.1371/journal.pone.0062211

4. Schaaf HS, Collins A, Bekker A, et al. Tuberculosis at extremes of age. Respirology 2010;15(5):747-763. https://doi.org/10.1111/j.1440-1843.2010.01784.x

5. Jana N, Vasishta K, Jindal SK, et al. Perinatal outcome in pregnancies complicated by pulmonary tuberculosis. Int J Gynecol Obstet 1994;44(2):119-124. https://doi.org/10.1016/0020-7292(94)90064-7
6. Peng W, Yang J, Liu E. Analysis of 170 cases of congenital TB reported in the literature between 1946 and 2009. Pediatr Pulmonol 2011;46(12):1215-1224. https://doi.org/10.1002/ppul.21490

Mittal H, Das S, Faridi MMA. Management of newborn infant born to mother suffering from

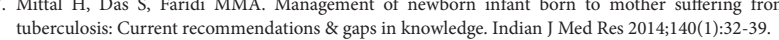

8. Schaaf HS, Nel ED. Tuberculosis presenting as cholestatic jaundice in early infancy. J Pediatr Schaaf HS, Nel ED. Tuberculosis presenting as cholestatic jaundice in early infancy. J
Gastroenterol Nutr 1992;15(4):437-439. https:// doi.org/10.1097/00005176-199211000-00012

Gastroenterol Nutr 1992;15(4):437-439. https:// doi.org/10.1097/00005176-199211000-00012
Marais BJ, Gie RP, Schaaf HS, et al. The natural history of childhood intra-thoracic tuberculosis: A critical review of literature from the pre-chemotherapy era. Int J Tuberc Lung Dis 2004;8(4):392-402.

10. National Department of Health, South Africa. Guideline for the prevention of mother to child transmission of communicable infections (HIV, hepatitis, listeriosis, malaria, syphilis and TB). 2019 https://www.nicd.ac.za/wp-content/uploads/2019/11/Guidelines-for-the-Prevention-of-Transmissionof-Communicable-Diseases-from-mother-to-child_28-October.pdf (accessed 25 January 2021).

11. World Health Organization. Latent tuberculosis infection: Updated and consolidated guidelines for programmatic management. Geneva: WHO, 2018. https://www.who.int/tb/publications/2018/latenttuberculosis-infection/en/ (accessed 25 January 2021).

12. World Health Organization. Guidance for national tuberculosis programmes on the management of tuberculosis in children. 2nd ed. WHO Press, 2014. https://www.who.int/tb/publications/childtb_ guidelines/en/ (accessed 25 January 2021)

13. Trébucq A. Should ethambutol be recommended for routine treatment of tuberculosis in children? A review of the literature. Int J Tuberc Lung Dis 1997;1(1):12-15.

14. Graham SM, Daley HM, Banerjee A, et al. Ethambutol in tuberculosis: Time to reconsider? Arch Dis Child 1998;79(3):274-278. https://doi.org/10.1136/adc.79.3.274

15. Du Preez K, Seddon JA, Schaaf HS, et al. Global shortages of BCG vaccine and tuberculous meningitis in children. Lancet Glob Health 2019;7(1):e28-e29. https://doi.org/10.1016/S2214-109X(18)30474-1

6. James JC, Nuttall, BS Eley. BCG vaccination in HIV-infected children. Tuberc Res Trea 2011;2011:712736. https://doi.org/10.1155/2011/712736

17. Bekker A, Schaaf HS, Draper HR, et al. Tuberculosis disease during pregnancy and treatment outcomes in HIV-infected and uninfected women at a referral hospital in Cape Town. PLoS ONE 2016;11(11):e0164249. https://doi.org/10.1371/journal.pone.0164249

18. McIlleron H, Meinties G, Burman WJ, et al. Complications of antiretroviral therapy in patients with tuberculosis: Drug interactions, toxicity and immune reconstitution inflammatory syndrome. J Infect Dis 2007;196(Suppl 1):S63-S75. https://doi.org/10.1086/518655

19. Bekker A, Schaaf HS, Seifart HI, et al. Antimicrob Agents Chemother 2014;58(4):2229-2234. https:// doi.org/10.1128/AAC.01532-13

20. Zenhäusern J, Bekker A, Wates MA, et al. Tuberculosis transmission in a hospitalised neonate: Need for optimised tuberculosis screening of pregnant and postpartum women. S Afr Med J 2019;109(5):310313. https://doi.org/10.7196/SAMJ.2019.v109i5.13789

21. Pillay T, Adhikari M. Congenital tuberculosis in a neonatal intensive care unit. Clin Infect Dis 1999;29(2):467-468. https://doi.org/10.1086/520250

22. Moore DP, Schaaf HS, Nuttall J, et al. Childhood tuberculosis guidelines of the Southern Africa Society for Paediatric Infectious Diseases. S Afr J Epidemiol Infect 2017;24:57-68.

23. Lee LH, LeVea CM, Graman PS. Congenital tuberculosis in a neonatal intensive care unit: Case report epidemiological investigation, and management of exposures. Clin Infect Dis 1998;27(3):474-477. https://doi.org/10.1086/514690

24. Seddon JA, Paton J, Nademi Z, et al. The impact of BCG vaccination on tuberculin skin test responses in children is age dependent: Evidence to be considered when screening children for tuberculosis infection. Thorax 2016;71(10):932-939. https://doi.org/10.1136/thoraxjnl-2015-207687

25. MacLean E, Sulis G, Denkinger CM, et al. Diagnostic accuracy of stool Xpert MTB/RIF for detection of pulmonary tuberculosis in children: A systematic review and meta-analysis. J Clin Microbiol 2019;57(6):e02057-18. https://doi.org/10.1128/JCM.02057-18 